\title{
Gestión interuniversitaria y responsabilidad en la gestión ambiental: Plan de acción para el mejoramiento de la gestión integral de los residuos sólidos
}

\author{
Inter-University Management: Action plan for the improvement of the integrated solid waste management \\ in the Guácimo Canton, Limón, Costa Rica
}

Recibido 01 de mayo 2014 • Aceptado 08 de diciembre 2014 • Corregido 02 marzo 2015

\author{
Rooel Campos Rodríguez \\ Docente-investigador \\ Escuela de Ingeniería de Agronegocios \\ Instituto Tecnológico de Costa Rica \\ Cartago, Costa Rica \\ rocampos@itcr.ac.cr \\ María Marta Camacho Álvarez² \\ Directora Escuela de Formación Docente \\ Universidad de Costa Rica \\ San José, Costa Rica \\ mariamarta.camacho@ucr.ac.cr
}

\begin{abstract}
Resumen. El presente artículo reporta los principales hallazgos de una investigación en el campo de la educación ambiental como un aporte para la gestión interuniversitaria. Es un estudio que, en su ejecución, contó con la participación de tres universidades estatales del sistema educativo costarricense: el Tecnológico de Costa Rica (TEC), la Universidad de Costa Rica (UCR) y la Universidad Nacional (UNA). El objetivo general se encauza hacia proponer un modelo ambiental para la adecuada gestión de los residuos sólidos en el cantón de Guácimo, ubicado en la provincia de Limón, Costa Rica y así, ofrecer insumos para la gestión universitaria en este campo. La investigación se realizó bajo un enfoque mixto; dentro de sus principales hallazgos se determinó que el problema ambiental de la región en estudio es multivariado, incluye aspectos de decisión política, dificultades administrativas, problemas de planeamiento, falta de inversión, inexistencia de planes de gestión, inexistencia de controles, así como baja cobertura en la recolección, ausencia de sitios apropiados para el tratamiento de residuos y poca formación en el área de la educación y la salud ambiental, aspectos que se tratan de atender desde la gestión inter, multi y transdisciplinar, así como interuniversitaria.
\end{abstract}

1 Doctor en Ciencias Naturales para el Desarrollo con énfasis en Gestión y Cultura Ambiental. Posee Maestría en Gestión Ambiental y Bachillerato en Ingeniería Agropecuaria Administrativa del Instituto Tecnológico de Costa Rica (ITCR). Actualmente se desempeña como coordinador del Área Académica Agroforestal y es profesor e investigador de la Escuela de Agronegocios del Tecnológico de Costa Rica.

2 Doctora en Educación con énfasis en Administración Educativa de la Universidad Latina de Costa Rica. Posee una Licenciatura en Educación Preescolar y un Bachillerato en Ciencias de la Educación con énfasis en Educación Preescolar de la Universidad de Costa Rica (UCR). Actualmente se desempeña como Directora de la Escuela de Formación Docente de la Facultad de Educación de la Universidad de Costa Rica (UCR). 
Palabras clave. Gestión interuniversitaria; educación ambiental; residuos sólidos; modelo ambiental; plan de acción para mejoramiento; redes de apoyo; procesos comunales; gestión residuos sólidos

\begin{abstract}
This article reports the main findings of a research conducted in the field of environmental education, as a contribution to inter-university management. Three state universities that are part of the Costa Rican educational system participated in the execution of this study: Instituto Tecnológico de Costa Rica (TEC), Universidad de Costa Rica (UCR) and Universidad Nacional (UNA). The general objective aims at proposing an environmental model for adequate solid waste management in Guácimo canton, located in the province of Limón, Costa Rica, and offering inputs for university management in this field. The research was carried out following a mixed approach, and one of the main findings indicates that the environmental issue in the region under study is multivariate, including aspects such as politics, administrative difficulties, planning problems, lack of investment, absence of management plans and controls, as well as low coverage of collection activities, absence of appropriate places for treatment of waste and few training opportunities in the area of environmental education and health. These aspects are addressed from the inter, multi, transdisciplinary and interuniversity management viewpoints.
\end{abstract}

Keywords. Interuniversity management; environmental education; solid waste; environmental model; action plan for improvement; support networks; communal processes; solid waste management

\title{
Introducción
}

Los distintos desafíos que enfrenta la sociedad según las demandas emergentes, inciden en ámbitos tales como educativo, económico, social, político y cultural; todos ellos interconectados respondiendo a las necesidades que, desde las estructuras sociales, se espera de ellos. A la educación superior, por su propia naturaleza, le corresponde asumir diferentes retos, como una de las instancias responsable de liderar los cambios que se esperan en busca del desarrollo económico y humano que se aspira en los diferentes contextos.

Siendo la administración universitaria el medio por el cual se operacionalizan las distintas políticas institucionales y sociales, debe actuar con un enfoque integral de todos los espacios de los que está conformada la sociedad. La administración universitaria orienta sus esfuerzos y recursos para atender la diversidad de demandas; una de ellas es la atención a la problemática de los residuos sólidos, en la cual la gestión universitaria tiene un papel estratégico, tanto en propuesta de administración como en procesos formativos en este ámbito.

Es en esta coyuntura, que el presente artículo obedece a una investigación sobre la gestión integral de los residuos sólidos desde la administración universitaria, tomando en cuenta los cinco ejes estratégicos del Plan nacional de la educación superior universitaria estatal (PLANES) 2011-2015, que representan pilares fundamentales para el proceso de investigación, docencia y acción social, así como sus objetivos: 
1. Potenciar la integración de las universidades estatales a fin de promover la excelencia en las transformaciones que requiere la sociedad costarricense.

2. Fortalecer los espacios estratégicos creados entre las universidades estatales para el análisis, evaluación y desarrollo de programas y proyectos conjuntos e innovadores en la formación de profesionales de alto nivel, generación de conocimiento y creación de nuevas oportunidades para el desarrollo de la nación, de acuerdo con las prioridades establecidas.

3. Consolidar la cooperación interinstitucional bajo el principio de integralidad, para optimizar la calidad del quehacer académico.

4. Potenciar las fortalezas complementarias de las universidades para elevar el impacto de su acción y responder a las demandas de la sociedad. (Consejo Nacional de Rectores [CONARE], 2011, p. 2)

Tomando en cuenta que un modelo ambiental es, según Wegener (2000), una representación simplificada de un objeto de investigación, es que este estudio se formuló con una visión sistémica e integradora, entendiendo sistema como un conjunto estructurado de componentes y variables que muestran relaciones entre ellos y operan en conjunto (Chorley y Kennedy, 1971), razón por la cual este análisis desarrolla un plan de acción sustentado en un modelo incorporado; abarcando aspectos de los medios ambientales, sociales, geográficos, sociológicos, económicos, políticos, culturales, recreativos y educativos.

\section{Sustento teórico}

\section{Gestión universitaria}

Pueden identificarse tres conceptualizaciones convencionales de la gestión. La primera, referida a la captación, ordenamiento, afectación y empleo de los recursos necesarios para el cumplimiento de los fines organizacionales que tienden a reducir el análisis a las funciones administrativas clásicas: planificación, organización, dirección, coordinación, control y gestión de recursos. La segunda, incorpora las dimensiones vinculadas al gobierno y la capacidad para definir el diseño institucional, las políticas que regulan su funcionamiento, la orientación estratégica, los objetivos operacionales, las ofertas académicas, los servicios, los estándares de calidad, así como el seguimiento y la evaluación de la ejecución de las acciones. La tercera, identifica los factores intervinientes que permiten explicar las capacidades de orientación y conducción institucional, así como las predisposiciones para percibir, atender y encauzar la creciente complejidad en que se debate la institución universitaria. Cada una de las conceptualizaciones anteriores aportan elementos claves a la gestión universitaria, aunque vistas por separado muestran sus posibilidades pero también sus carencias; por lo tanto, realizar 
un proceso más holístico requiere retomar los elementos relevantes de cada una de ellas y darles un sentido más integral e integrador (Masten, 2000; Martínez y Góngora, 2000).

De acuerdo con los autores mencionados en el párrafo anterior, la gestión institucional suele ser entendida como la resultante de la agregación de las gestiones específicas de las funciones aludidas o por otra parte, como la administración de todos los recursos a disposición de la universidad, sean ellos materiales, financieros o humanos. Sin embargo, la gestión universitaria trasciende; es un proceso integral de apoyo para la toma de decisiones en las actividades sustantivas de las instituciones. Se caracteriza por la búsqueda del mejoramiento continuo, para lo cual contempla el desarrollo de procesos innovadores y el uso de tecnologías de información y comunicación en un marco de transparencia, de rendición de cuentas y de transformación social.

De acuerdo con CONARE (2011), el avance científico y tecnológico ha colocado a Costa Rica en una posición de liderazgo en Centroamérica y el Caribe en materia de investigación, desarrollo e innovación. Las universidades estatales han desempeñado un papel decisivo en los logros alcanzados en estos espacios, tanto en el apoyo coyuntural en materia de docencia, así como en el compromiso con la sociedad a través de la investigación, la extensión y la acción social, pues debe conducir al desarrollo integral de las instituciones y procurar la transformación social en todas las dimensiones posibles.

\section{Modelo de educación ambiental y gestión universitaria}

Las universidades, desde sus áreas de docencia, investigación y acción social, desarrollan planes, estrategias, proyectos y acciones relacionadas con el tema ambiental, en algunos casos como ejes estratégicos y en otros, como ejes transversales.

El Plan nacional de la educación superior universitaria estatal (PLANES) 2011-2015, propuesto por CONARE (2011), presenta cinco ejes estratégicos: pertinencia e impacto, acceso y equidad, aprendizaje, ciencia y tecnología y finalmente, gestión. En este marco de planificación se define como objetivo estratégico: Vinculary gestionar el quehacer universitario con acciones orientadas al desarrollo sostenible (social, biológico, físico). Para este eje se han determinado tres acciones relevantes: en primer lugar, fortalecer programas y proyectos de docencia, investigación, vida estudiantil, extensión y acción social, así como de gestión universitaria, orientados a impulsar el desarrollo sostenible; en segundo lugar, consolidar el tema ambiental en los programas académicos para formar profesionales con una clara conciencia de su responsabilidad en ese ámbito $y$, en tercer lugar, apoyar planes, programas y proyectos en temas estratégicos con implicaciones ambientales, en coordinación con los diversos sectores nacionales.

Al respecto, cabe resaltar que existe una amplia gama de modelos ambientales, pero como señalan López, López y Rodríguez (2005), los modelos deben enfocarse en la sostenibilidad de las comunidades, haciendo un análisis de su problemática socioambiental, con programas que 
DOI:

URL: http://revistas.ucr.ac.cr/index.php/gestedu

integren la educación, la cultura, la producción y conservación, con el fin de coadyuvar a la mejora de las condiciones socioeconómicas por medio de un manejo sostenible de los recursos naturales; de forma tal, que la problemática ambiental que se aborde sea con conocimiento del medio social, ambiental, económico y cultural para que las acciones sea integrales. En ese sentido, Caride y Meira (2001) indican que la educación ambiental es la que permite aproximarse a un desarrollo ecológicamente sostenible y humanamente equitativo.

Los modelos de educación ambiental se pueden ver desde dos perspectivas: primero, como una tecnología social, cuyo fin primordial es la minimización de los impactos ambientales negativos al medio y la otra, que pretende un cambio global de la humanidad hacia una civilización socialmente más justa y ecológicamente sostenible. En la actualidad no existe un modelo definitivo ni enfoques que puedan resolver todas las problemáticas ambientales, por lo que si se espera un cambio ambiental, primero debe darse un cambio social y educativo, para que las estrategias y planes ambientales conlleven a cambios voluntarios y conscientes que perduren en el tiempo y con el involucramiento de diferentes actores (Gutiérrez y Pozo, 2006).

De acuerdo con la Conferencia Mundial de Educación Superior de la Organización de las Naciones Unidas para la Educación, la Ciencia y la Cultura (UNESCO, 2009, p. 6): “Los establecimientos de enseñanza superior deberían buscar esferas de investigación y docencia capaces de abordar los asuntos que atañen al bienestar de la población y crear bases sólidas para la ciencia y la tecnología pertinentes en el plano local". Por su parte, en la Conferencia Regional de Educación Superior en América Latina y el Caribe (CRES) realizada por el Instituto Internacional de la UNESCO para la Educación Superior de América Latina y el Caribe (UNESCOIESALC, 2008) se determina que:

En un mundo donde el conocimiento, la ciencia y la tecnología juegan un papel de primer orden, el desarrollo y el fortalecimiento de la Educación Superior constituyen un elemento insustituible para el avance social, la generación de riqueza, el fortalecimiento de las identidades culturales, la cohesión social, la lucha contra la pobreza y el hambre, la prevención del cambio climático y la crisis energética, así como para la promoción de una cultura de paz. (p. 134)

Visto de esta manera, los modelos ambientales deben centrarse en prácticas educativas que logren compensar las necesidades de conocimiento y formación, tal como lo proponen López et ál. (2005), que busquen ampliar la gama de opciones de la sociedad con miras a mejorar la calidad de vida a partir de la solución de problemas cotidianos, así como la satisfacción de necesidades capitales, dado que el logro de mejores niveles de vida, más oportunidades de formación y adquisición de capacidades influyen en las personas y por lo tanto, en los procesos de transformación y mejora social.

A nivel general, algunos modelos ambientales son: a) Teoría de Conducta Planificada (Ajzen, 1985), en donde seguir o no seguir una conducta dependerá de la intención de la persona 
de hacerlo, de variables de actitud, normas subjetivas y control percibido del comportamiento; b) Comportamiento Ambiental Responsable (Hungerford y Volk, 1990) y (Hsu, 2004) que pretende predecir el comportamiento ambiental basándose en variables de entrada, apropiación y empoderamiento; c) Acción Positiva (Emmons, 1997), el cual enfatiza más en la acción que en el comportamiento, en donde se interrelacionan los conceptos ambientales, las habilidades, actitudes y el empoderamiento para llegar a una acción ambiental positiva; d) Predicción del comportamiento ambiental a partir de actitudes (Tarrant y Cordell, 1997) que pretenden resaltar la importancia de las variables personales y las situacionales para llegar a un comportamiento ambiental.

En Costa Rica se han realizado esfuerzos valiosos orientados a líderes comunales. Sin embargo, estos modelos, en términos generales, han tenido un escollo importante por la falta de participación de las comunidades, lo que incide directamente en la no solución de los problemas ambientales; no obstante, los modelos que han logrado una participación más activa de la comunidad desde su etapa de planificación, han obtenido logros importantes y un efecto positivo en el comportamiento ambiental de los participantes (Guier, Rodríguez y Zúñiga, 2004).

Para una gestión integral de residuos sólidos en Costa Rica no solo es suficiente contar con elementos técnicos y legales; es necesaria además, la incorporación de profesionales en diversos campos, así como una sociedad informada y sensibilizada. Con lo anteriormente referido, se podría obtener lo siguiente, sustentado en el Programa Competitividad y Medio Ambiente (CYMA, 2008, p. 58), el cual hace referencia a que: "Una población que realiza un consumo responsable y participa activamente en las propuestas de Gestión Integrada de Residuos Sólidos; instituciones públicas que son reflejo de un Estado comprometido con la GIRS y agrupaciones sociales participando con iniciativas y programas específicos".

La educación ambiental, además de abarcar la parte teórica, debe procurar que desde las mismas comunidades emanen las alternativas de solución de las problemáticas ambientales por medio de modelos educacionales enfocados en el desarrollo sostenible. Ejemplo de ello es la planificación, desarrollo y evaluación del plan de acción para el mejoramiento de la gestión integral de los residuos sólidos en el cantón de Guácimo, propuesta elaborada y desarrollada de manera interuniversitaria, la cual se explicará con mayor amplitud en los siguientes párrafos.

\section{Sustento metodológico}

El objetivo general que orienta el desarrollo de la presente investigación es proponer un modelo ambiental para la adecuada gestión de los residuos sólidos en el cantón de Guácimo, Limón, Costa Rica. Dentro de sus objetivos específicos, en este artículo se consideran: a) Realizar un estudio sobre conocimientos, habilidades, actitudes y barreras según los actores sujetos del estudio; y b) Diseñar un modelo ambiental de líneas de para la gestión integral de este tipo de residuos, que involucre a los actores sociales. 
Esta investigación se realizó bajo un enfoque mixto, para lograr una perspectiva más amplia, integral y completa de la situación en estudio. De esta forma, es posible obtener mayor cantidad de perspectivas en cuanto a magnitud (cuantitativo) y profundidad (cualitativo), en especial considerando que los problemas ambientales son complejos y se necesita un entendimiento comprehensivo de la dinámica social, ambiental, cultural y económica para poder describir, analizar y explicar la situación de los residuos sólidos (Creswell, 2005). El estudio es de tipo descriptivo y explicativo.

Para el análisis cuantitativo de la información, se utilizó una hoja de cálculo, con el fin de tabular y obtener los resultados en cuanto a las cantidades de residuos sólidos que se generaron. De esta forma, los datos se ordenaron en cuadros y figuras resumen que reflejaron los valores de los indicadores sobre la generación de residuos sólidos, así como la composición. Para el análisis cualitativo se realizaron entrevistas semiestructuradas, con el fin de recabar información de primera mano; se hicieron visitas para corroborar la información contenida en las entrevistas. Con todos estos insumos se analizó la información y se establecieron algunos sustentos teóricos-prácticos, tendencias y estimaciones.

Para determinar los conocimientos, comportamientos, habilidades, actitudes y barreras de los participantes del objeto de estudio, se aplicó una encuesta a una muestra representativa de los sujetos informantes, las cuales fueron tabuladas, graficadas y analizadas de manera descriptiva y explicativa, construyendo categorías para el análisis de la información de tipo cualitativa. A partir de los resultados, se propuso el plan de acción para el mejoramiento de la gestión integral de los residuos sólidos en el cantón de Guácimo.

\section{Escenario y características de la población}

Para realizar la investigación se escogió el cantón de Guácimo, porque presenta un índice de desarrollo social (IDS) de 47,8, el cual es uno de los más bajos del país y se encuentra adicionalmente, en la provincia con mayor rezago social y económico, con problemas de pobreza, deterioro de ecosistemas y servicios públicos (Programa de las Naciones Unidas para el Desarrollo [PNUD] y Proyecto de Fortalecimiento Municipal y Descentralización [FOMUDE], 2009). El 73\% de la población se concentra en el centro urbano (distritos de Guácimo y Jiménez), y solo a estos sectores se les brinda el servicio de recolección municipal de residuos sólidos, el resto de población del cantón no recibe este servicio por pertenecer a zonas rurales poco densificadas.

Para el alcance de los objetivos segundo y cuarto de la investigación, se contó con una población de cuarenta personas representantes de las siguientes instancias: Concejo, personal administrativo y de Saneamiento Ambiental de la Municipalidad de Guácimo, Comité PRESOL (Programa de Residuos Sólidos), Ministerio de Salud, Ministerio de Educación (por medio de instituciones de educación preescolar, primaria y media), representantes de Centros de 
Recuperación de Materiales recolectores de residuos sólidos de Guácimo, sector comercial así como un grupo piloto de viviendas de la comunidad.

\section{Resultados y análisis del proceso de implementación de las diversas fases}

De manera general, cabe apuntar que la situación actual del cantón de Guácimo lo coloca en una posición de alta vulnerabilidad en el manejo de los residuos sólidos, ya que el problema es multivariado, pues incluye aspectos de decisión política, dificultades administrativas, problemas de planeamiento, falta de inversión, inexistencia de planes de gestión, baja cobertura de recolección, ausencia de sitios de tratamiento apropiados y poca formación en el área de la educación y la salud ambiental.

A nivel técnico operativo, el municipio parte de los estudios de generación y composición realizados por Campos (2014), estos evidenciaron la existencia de un índice de generación

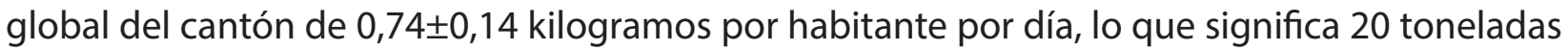
diarias de residuos. El municipio cuenta con un Plan cantonal para el desarrollo humano local de Guácimo (PNUD-FOMUDE, 2009), donde una de sus políticas generales se centra en el área de gestión ambiental, con énfasis en garantizar un manejo integral de los desechos sólidos en cumplimiento de la ley y las normas ambientales. Sin embargo, en el cantón existe la problemática del botadero municipal que debe ser cerrado definitivamente, ya que existe la orden de cierre sanitario por parte del Ministerio de Salud. Por otra parte, la municipalidad de la localidad actúa conforme a las urgencias, solicitudes y demandas, por lo que no optimizan sus recursos, sobrecargan al personal, resuelven problemas de manera atomizada y con pocos recursos. Además de esto, la escasa o nula capacitación del personal de saneamiento ambiental no permite un aprovechamiento de las habilidades y destrezas de estos colaboradores, sumado a la escasez de presupuesto para desarrollar las labores.

Al tomar en cuenta los conocimientos previos que tiene la población en estudio sobre educación ambiental y residuos sólidos, estos muestran que es: "Proceso que educa en la manera cómo cuidar el ambiente para mejorar la calidad de vida"; "Va dirigida al conocimiento, al uso adecuado de los recursos, a la preservación del medio, a crear conciencia responsable"; "Conocimiento para aprovechar los recursos naturales para reciclar, crear Centros de Acopio, aprovechar material reciclado para artesanía. Proteger los ríos, la flora y la fauna". "Educación Ambiental es todo lo que tiene que ver con el cuidado del medio ambiente. Ejemplo: ríos, bosques, recursos naturales"; "Tiene que ver con la limpieza, naturaleza, reciclaje. Conocimiento para futuras generaciones. Proceso de enseñanza y aprendizaje de cómo convivir con la naturaleza"; "Proceso de enseñanza por el cual darle un adecuado manejo a los desechos para así tener una mejor calidad de vida".

Vinculadas con la definición de residuos sólidos, algunas de las respuestas indicadas por los participantes fueron: "Están presentes en cualquier proceso, en gran parte son reutilizables y una 
DOI:

URL: http://revistas.ucr.ac.cr/index.php/gestedu

pequeña porción son desechos"; "Es todo lo que se puede aprovechar: plásticos, aluminio, cartón, vidrio, y orgánicos"; "Es todo material que no se desintegra, se puede reutilizar. Se aprovechan en diferentes usos, ejemplo: drenajes con llantas; plástico para floreros; cartón para jarrones. Además, generan contaminación, pocos residuos sólidos son aprovechables, muchos son desechados. Algunos residuos sólidos son reciclables y otros solo son reutilizables"; "Corresponde a los productos que se consumen diariamente. Es una fuente de empleo en el cantón". "Objetos que ya no se utilizan y se pueden reutilizar. Ejemplo: botella, hojas, cáscaras".

Partiendo del hecho de que la educación ambiental se concibe como un proceso inherente a todo espacio educativo, conducente a la formación de personas conscientes de que pertenecen a un sistema ecológico global, regido por una serie de leyes y principios que deben conocerse y sobre todo respetarse para garantizar la vida misma, cabe destacar que la noción que tiene el grupo piloto sobre este tema es pertinente y hace ver la sensibilidad y el empoderamiento alcanzado a lo largo del proceso de investigación.

Por otra parte, los participantes señalaron que cuentan con el apoyo de las Universidades (TEC, UNA, UCR, Universidad EARTH) en los procesos que se desarrollan en el cantón de Guácimo, y que poseen algunos conocimientos en los temas tratados, reforzados con apoyo de la academia. Asimismo, advierten que se desarrollan varios proyectos a nivel escolar, además de la existencia de diversas iniciativas comunales que evidencian la relación entre lo teórico y lo práctico con acciones y proyectos tales como cooperativas o centros de acopio para separar los desechos sólidos y organizaciones que reciclan. Por otra parte, expresan que se cuenta con el apoyo de los trabajadores de la municipalidad (sanidad ambiental).

En el ámbito del talento humano, apuntan que hay personas comprometidas y buenos líderes comunales, designando también que varias personas usan residuos para crear artesanía, pero sobre todo indican que hay disposición al cambio.

En el ámbito local expresan que existe compromiso del gobierno local en protección del recurso hídrico y en el manejo de residuos sólidos, integración de grupos y buena disposición por parte de varias instituciones públicas que apoyan. Así mismo, recalcan que falta transporte e infraestructura para una mejor disposición y tratamiento de los residuos sólidos.

De acuerdo con el grupo participante, las barreras presentes para un manejo adecuado de los residuos sólidos se pueden resumir de la siguiente forma:

- En el ámbito de gobernabilidad local señalan que, entre otras cosas, hace falta el interés de altos jerarcas del municipio, recursos para el manejo apropiado de los residuos, un plan adecuado de manejo, un sistema de cobro por tonelaje, un centro de acopio y equipo que dé continuidad al proyecto, espacio para acopio, maquinaria para recolección, y depósitos en la vía pública del cantón que permita separar residuos.

- En el ámbito educativo se asevera que no existen campañas de educación ambiental ni 
de concientización a la población; así como la inexistencia de una cultura de manejo de residuos. Además, subrayan que hay corrupción e incumplimiento de deberes, así como desconocimiento.

- En el ámbito actitudinal, en general, se señala la falta de compromiso y la presencia de personas reacias al cambio, así como poco interés y poca motivación por parte de la comunidad. Comentarios como: "no tengo tiempo". "no me interesa", "no tengo espacio", "no sé cómo clasificarlo", "no tengo dónde dejarlo" "no me gusta" son algunos ejemplos comunes.

Los retos que los participantes del proceso mencionan son transcritos literalmente a continuación: "llevar la información a la comunidad para que aprenda a salvar la naturaleza, seguir trabajando con las comunidades para futuro, llevar motivación e información, hacer cambio para un mejor desarrollo del cantón, aprovechar los conocimientos adquiridos y sacar provecho de los contactos estratégicos establecidos, que este curso pueda llegar a más personas para alcanzar una mayor sensibilización, poder demostrar la efectividad de conocimiento del curso, mediante la práctica, integrar un mayor número de participantes que se comprometan al medio ambiente, pasar la información a la familia y comunidad donde trabajamos, concientizar a la familia para que reflexione y cuide mejor la calidad de vida, mayor participación de las personas jóvenes, hacer que tomen conciencia las instituciones municipales, concientizar a la población y llevar nuevas estrategias a la comunidad y continuar con estos proyectos en más comunidades o cantones de nuestro país".

Para generar cambios en los conocimientos, transformar las barreras y los retos expuestos, el Tecnológico de Costa Rica, la Universidad Nacional y la Universidad de Costa Rica se sumaron para abordar el tema desde las tres áreas sustantivas: las áreas de docencia e investigación se impulsaron bajo la coordinación del TEC, con el apoyo de la UCR y la UNA; por otra parte, la acción social se coordinó desde la UCR, con el apoyo del TEC y la UNA. Cabe destacar que todos los procesos se realizaron de manera integrada; se hace esta aclaración debido a que los procesos de trabajo interuniversitario en Costa Rica se han desarrollado en los últimos años desde dos visiones muy diferentes: por un lado, universidades que presentan proyectos interuniversitarios, pero que cada una administra por separado, en regiones geográficas distintas y solo con un enlace de coordinación; y por otro lado, universidades que estrechan más sus vínculos y que coordinan todos sus procesos, siendo este el caso de la relación que se promovió para este proyecto, donde se contó con la participación de representantes de las tres universidades en cada una de las fases del plan de acción. Cabe destacar que entre los colaboradores universitarios se incorporó a personal docente, administrativo y estudiantil. Al integrar a la población estudiantil se cumple con uno de los objetivos primordiales de las universidades que es lograr la participación activa de los estudiantes en la vida académica, garantizándoles la libertad de expresión y el derecho a organizarse; así como con los acuerdos tomados en la Conferencia Mundial de la Educación Superior (UNESCO, 2009, p. 2) la cual indica: 
La educación superior debe no solo proporcionar competencias sólidas para el mundo de hoy y de mañana, sino contribuir además a la formación de ciudadanos dotados de principios éticos, comprometidos con la construcción de la paz, la defensa de los derechos humanos y los valores de la democracia.

\section{Diseño de un plan de acción para el mejoramiento de la gestión integral de los residuos sólidos, que involucre a los actores sociales del cantón de Guácimo}

En la Conferencia Regional de Educación Superior de América Latina y el Caribe (UNESCOIESALC, 2008) se declara que:

Las instituciones de Educación Superior deben avanzar en la configuración de una relación más activa con sus contextos. La calidad está vinculada a la pertinencia y la responsabilidad con el desarrollo sostenible de la sociedad. Ello exige impulsar un modelo académico caracterizado por la indagación de los problemas en sus contextos; la producción y transferencia del valor social de los conocimientos; el trabajo conjunto con las comunidades; una investigación científica, tecnológica, humanística y artística fundada en la definición explícita de problemas a atender, de solución fundamental para el desarrollo del país o la región, y el bienestar de la población; una activa labor de divulgación, vinculada a la creación de conciencia ciudadana sustentada en el respeto a los derechos humanos y la diversidad cultural; un trabajo de extensión que enriquezca la formación, colabore en detectar problemas para la agenda de investigación y cree espacios de acción conjunta con distintos actores sociales, especialmente los más postergados. (p. 7)

Siguiendo la línea de integración e influencia de la academia en beneficio de la sociedad citada anteriormente, se conformó un plan de acción para el mejoramiento, cuyas fases fueron aplicadas en la comunidad de Santa María de Guácimo, desde el año 2011. Para el perfeccionamiento de un plan de acción se considera importante no solo la fase de planificación y desarrollo, sino también el seguimiento y el acompañamiento a los actores involucrados para que puedan sensibilizarse, interiorizar los conocimientos y empoderarse y así, puedan continuar desarrollando los distintos objetivos, actividades y proyectos.

El plan de acción realizado de manera interinstitucional fue organizado por fases. La primera de ellas consistió en el proceso de diagnóstico e investigación de la situación, la cual fue realizada por el investigador principal con el apoyo de actores sociales de la comunidad local y las comunidades universitarias, quienes a su vez se conforman en una red para estudiar, comprender y buscar soluciones al problema. De la fase de investigación se determinan retos que había que fortalecer en el proceso, siendo uno de ellos acerca de información y capacitación, por lo que se organizan dos fases complementarias: una de ellas la publicación de un manual de residuos sólidos y la otra, un proceso de capacitación por medio de un taller denominado Líneas 
de generación y aplicación del conocimiento para la Gestión Integral de los Residuos Sólidos del cantón de Guácimo en el que participaron representantes activos de instituciones involucradas con la temática en estudio. Como producto de las fases anteriores surgen dos fases adicionales, la de aplicación de plan de acción y la divulgación de resultados.

Para una mejor comprensión de las fases, estas se describen a continuación:

\section{Objetivo 4. Modelo Ambiental para la Gestión Integral de los Residuos Sólidos}

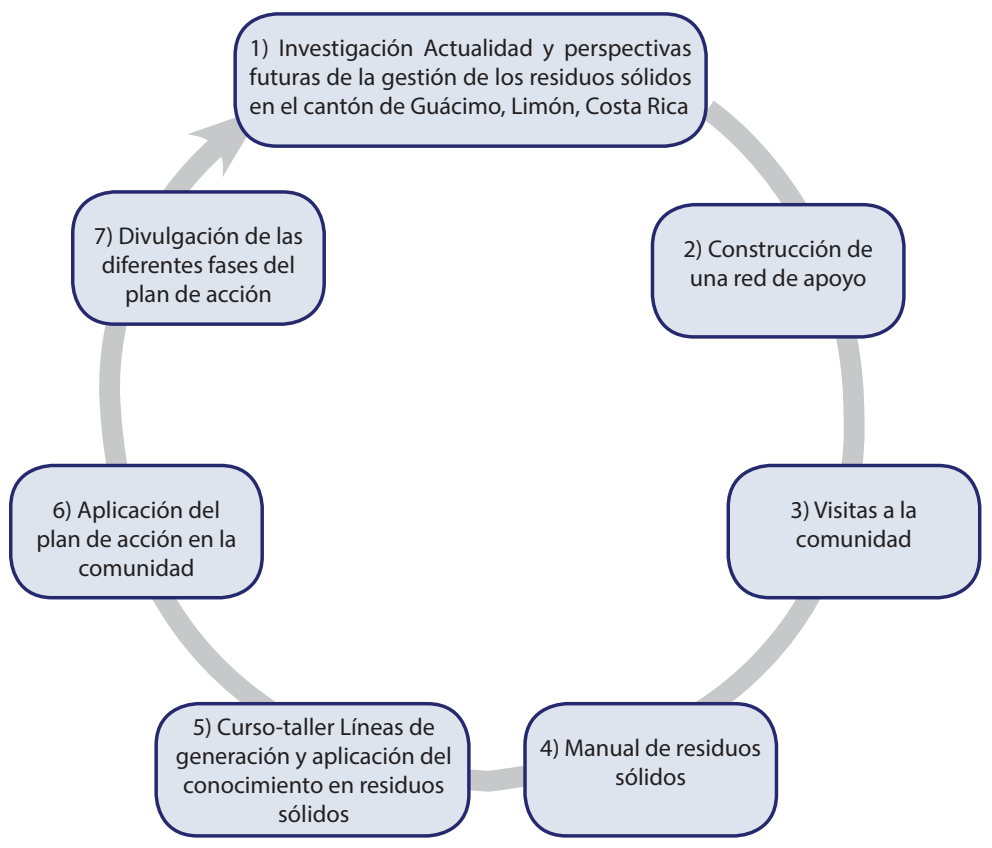

Nota: Campos, 2014.

Figura 1. Fases del Plan de acción para el mejoramiento de la gestión de residuos sólidos

Con este proceso se trata de abordar la situación problemática con un enfoque más integral, en el que la participación comunal e interuniversitaria se unen para generar un proceso continuo de cambio, del que se espera el empoderamiento y la participación de los actores y líderes comunales, así como el acercamiento de la teoría y la práctica en el ámbito universitario. 
Fase 1. Investigación Actualidad y perspectivas futuras de la gestión de los residuos sólidos en el cantón de Guácimo, Limón, Costa Rica

Con base en el eje Ciencia y tecnología, expresado en PLANES (CONARE, 2011), uno de los objetivos fundamentales está orientado a la investigación, la gestión de tecnologías de información-comunicación, así como la difusión y uso del conocimiento. Se propone que la investigación se vincule estrechamente con las necesidades de desarrollo de los sectores sociales y productivos, para que la responsabilidad social sea su principal criterio orientador.

Con respecto al eje mencionado, el plan de acción para el mejoramiento que se expone en este artículo permite el logro de los siguientes objetivos de PLANES, a saber: consolidar la investigación orientada hacia la innovación, para que contribuya con las transformaciones que requiere la sociedad, así como contribuir con el desarrollo nacional, mediante la difusión, intercambio y transferencia del conocimiento científico y tecnológico generado por las comunidades universitarias (CONARE, 2011).

En este caso, el trabajo de investigación analiza la generación y composición de residuos sólidos producto del aumento de la población y la industrialización como uno de los principales problemas ambientales de actualidad en la sociedad. Se determinó que el municipio no dispone de suficiente cantidad de información básica para implementar su sistema de gestión integral de residuos sólidos (GIRS), se logró indagar sobre los conocimientos, habilidades, actitudes y barreras del área de estudio, según los criterios de representantes del sector vivienda, comercios y la parte política de la Municipalidad. Además, se establecieron los índices de generación por habitante y la composición porcentual de residuos sólidos según los tipos de materiales definidos en la legislación costarricense.

En esta primera fase, el Tecnológico de Costa Rica lideró el proceso investigativo. La función de las personas involucradas de la Universidad de Costa Rica y de la Universidad Nacional fue colaborar con la parte práctica y de seguimiento de la investigación. El punto de partida fundamental del proyecto para los representantes de las universidades participantes está basado en la investigación, pues pretende generar cambios a partir de los resultados logrados así como de las expectativas y posibilidades comunales.

Con respecto a esta fase, la UNESCO, en sus conferencias, ha declarado la necesidad de facilitar estructuras para el diálogo y el intercambio de experiencias e informaciones sobre la educación superior y la investigación; que se debe promover la ciencia y la interdisciplinariedad, al servicio de la sociedad; la importancia que el personal docente universitario disponga de oportunidades para realizar investigaciones y obtener becas; la necesidad de facilitar estructuras para el diálogo y el intercambio de experiencias e informaciones sobre la educación superior y la investigación; así como la necesidad de disponer de más financiamiento para la investigación y el desarrollo, por medio de iniciativas conjuntas de múltiples copartícipes entre el sector público y el privado. 


\section{Fase 2. Construcción de una red de apoyo al proyecto}

Para que el proyecto obtuviera el éxito esperado, paralelo al proceso de investigación se organizó una red en donde fueron incorporadas la mayor cantidad de actores sociales de la comunidad, con el fin de generar un efecto multiplicador respecto a la gestión de los residuos sólidos en el cantón. También, se estableció un vínculo de colaboración con personal docente e investigadores del TEC, la UCR y la UNA, representantes administrativos de la Municipalidad de Guácimo, el Comité PRESOL (obligatorio por ley para todos los municipios costarricenses), el personal docente y administrativo de diversos centros educativos del cantón, así como su Junta Educativa, representantes de Canal 30 de televisión local, el Ministerio de Seguridad Pública, el Ministerio de Salud, el Comité Cruz Roja y el Comité de Deportes, lo que generó un mayor impacto a nivel cantonal para realizar un trabajo colaborativo respecto del tema de educación ambiental en general, y el manejo de residuos sólidos en particular.

Esta red de apoyo permitió ampliar los conocimientos, la participación activa y comprometida de la comunidad, aumentar las capacidades para la solución del problema de los residuos sólidos, el trabajo conjunto entre el sector político, social y educativo, así como el desarrollo de responsabilidades por parte de los actores involucrados, características que, en síntesis, resumen los objetivos del proceso de educación ambiental.

Al realizar esta fase, se cumple con uno de los ejes de PLANES (CONARE, 2011), denominado Pertinencia e Impacto, el cual tiene entre sus objetivos: fortalecer la vinculación de la educación superior universitaria estatal con los sectores sociales y productivos en los ámbitos nacional e internacional, para el mejoramiento de la calidad de vida; y como otro objetivo, vincular y gestionar el quehacer universitario con acciones orientadas al desarrollo sostenible (social, biológico, físico).

También se logra desarrollar algunos de los aspectos que se mencionan tanto en la Conferencia Regional de Educación Superior de América Latina y el Caribe (UNESCO-IESALC, 2008) como en la Conferencia Mundial de la Educación Superior (UNESCO, 2009), entre estos:

- Los establecimientos de enseñanza superior deberían buscar esferas de investigación y docencia capaces de abordar los asuntos que atañen al bienestar de la población y crear bases sólidas para la ciencia y la tecnología pertinentes en el plano local (UNESCO, 2009, p. 5).

- La Educación Superior tendrá que hacer efectivo el desarrollo de políticas de articulación con todo el sistema educativo, colaborando en la formación de sólidas bases cognitivas y de aprendizaje en los niveles precedentes, de tal manera que los estudiantes que ingresan al nivel superior cuenten con los valores, las habilidades, destrezas y capacidades para poder adquirir, construir y transferir conocimientos en beneficio de la sociedad. (UNESCO, 2008, p. 5) 
- Se necesita una educación que contribuya eficazmente a la convivencia democrática, a la tolerancia y a promover un espíritu de solidaridad y de cooperación; que construya la identidad continental; que genere oportunidades para quienes hoy no las tienen y que contribuya, con la creación del conocimiento, a la trasformación social y productiva de nuestras sociedades. (UNESCO, 2008, p. 3)

\section{Fase 3. Visitas a la comunidad}

Desde mediados del año 2011, se inició el contacto con la Municipalidad de Guácimo, dada la anuencia del Concejo, pues desde el primer acercamiento el ayuntamiento interiorizó la urgente necesidad de trabajar en el tema de la gestión de los residuos sólidos, se inició el trabajo con el comité PRESOL recabando la información necesaria para toda la logística que se debía seguir a nivel cantonal. Desde esa fecha, las reuniones presenciales, así como telefónicas, fueron semanales, con miembros de PRESOL, con la gestora ambiental y sobre todo, con el encargado del saneamiento ambiental del municipio. Se visitó a todos los distritos en sus poblados principales y especialmente, la comunidad de Santa María, lo que permitió establecer vínculos de colaboración con los diferentes actores sociales, un proceso de seguimiento, acompañamiento y credibilidad en el proceso.

Con respecto a la trascendencia de la gestión y los retos que implica, Martínez y Góngora (2000) apuntan que:

Un papel esencial de la gestión universitaria es la vigilancia de la coherencia entre su identidad, su orientación y su desempeño, reafirmando el sentido único al que deben responder las acciones, las respuestas a los desafíos contextuales y la resolución de las tensiones que enfrente y padezca. En consecuencia, la gestión no es una tecnología neutra ni una tarea de ingeniería: es una labor de construcción, preservación y proyección en el tiempo de los elementos que convierten a la organización en una institución que involucra a muy diversos actores y que encuentra su razón de ser en los fines a los que sirve y en los resultados que alcanza (p. 11)

Conocer la realidad desde el contacto con la comunidad permite observar, analizar, contrastar la realidad tanto a partir la teoría como desde la práctica. Transciende a las hipótesis que se formulan y confrontan a la comunidad y al equipo investigador con la realidad misma, con la finalidad de buscar procesos de transformación.

\section{Fase 4. Manual de residuos sólidos}

Como se ha explicado anteriormente, el manejo de los residuos sólidos se ha convertido en uno de los principales problemas ambientales de la sociedad actual, pues estos aumentan de manera proporcional con el crecimiento de la población y la industrialización. 
Con los resultados de la investigación se confeccionó un manual en el cual se explica la estrecha relación del sistema económico y la generación de residuos; es decir, el aumento poblacional implica mayores demandantes y oferentes de bienes y servicios, lo que deriva en una mayor posibilidad de generar residuos, que por no ser tratados adecuadamente se han convertido en un problema de interés local, nacional e internacional.

En este punto, se hace necesario comprender que la gestión integral de residuos pretende reconocer el valor intrínseco tanto económico como ambiental de los residuos sólidos, al abordar la problemática desde un enfoque sistémico. Un camino para alcanzar esto se puede dar, como apuntan Gómez y Reyes (2004), por medio de la creación de una conciencia ambiental, que derive en un cambio en los patrones de comportamiento de la sociedad, con el fin de brindar la oportunidad de contar con nuevos modelos que permitan el debate y la reflexión sobre el manejo actual de los recursos naturales. Por tal motivo, el propósito del manual es presentar información básica para el entendimiento de la comunidad sobre el tema de los residuos sólidos, así como el planteamiento de algunos de sus posibles usos alternativos, es decir, su valorización. Dicho documento tiene como base los hallazgos obtenidos a lo largo de la investigación.

Las fases cuatro y cinco del plan de acción descrito en este artículo, cumplen con uno de los ejes expuestos en PLANES (CONARE, 2011) denominado Aprendizaje, el cual señala como objetivo desarrollar y consolidar programas y proyectos de educación para toda la vida, que para el caso particular el manual y el taller se convierten en estrategias, recursos e insumos para enriquecer el conocimiento del área de educación ambiental en general y del manejo de residuos sólidos en particular.

Fase 5. Taller Líneas de generación y aplicación del conocimiento para la gestión integral de los residuos sólidos del cantón de Guácimo

\section{Línea estratégica educativa}

Como se ha mencionado a lo largo de este documento, para una gestión integral de residuos sólidos se hace necesaria la participación activa de las comunidades, por lo que es imperativa la promoción de instrumentos metodológicos que fortalezcan y promuevan la capacidad con que estas cuentan, para que tengan un control de los procesos, a partir de su propia idiosincrasia e interés de transitar por un cambio social, ambiental y cultural (López et ál., 2005).

De acuerdo con la Conferencia Regional de Educación Superior de América Latina y el Caribe (UNESCO-IESALC, 2008, p. 7): "Las instituciones de conocimiento tienen un papel fundamental en la orientación de las nuevas tecnologías y la innovación hacia sistemas de consumo-producción que no condicionen las mejoras en el bienestar al consumo creciente de energía y materiales". 
Por este motivo, la educación ambiental, además de preparar la documentación teórica, debe dar un paso más y procurar que desde las mismas comunidades emanen las alternativas de solución de las problemáticas ambientales por medio de planes enfocados en el Desarrollo Sostenible. Visto de esta manera, los planes ambientales deben enfocarse a prácticas educativas que logren compensar las necesidades de conocimiento y formación, con el objetivo de ampliar la gama de opciones de la sociedad y así, mejorar la calidad de vida a partir de la solución de problemas cotidianos y la satisfacción de necesidades capitales. En otras palabras, el logro de mejores niveles de vida, así como la consecución de más oportunidades de formación y adquisición de capacidades, son elementos que calarán en los individuos para que se den procesos de transformación y mejora social.

Por tal motivo, la línea estratégica educativa de este plan de acción se dirigió a un trabajo de capacitación en la comunidad de Santa María de Guácimo, tomando como base que la adquisición o redefinición de conocimientos por parte de los actores involucrados influye en las actitudes, habilidades, destrezas; coadyuvando a su vez, a la superación de barreras, permitiendo el empoderamiento.

El plan de capacitación, diseñado tipo curso-taller, tuvo como objetivo general analizar las líneas de generación y aplicación del conocimiento en educación ambiental, comunicación, sistemas de producción, conservación, tratamiento y disposición adecuada de residuos sólidos, que llevaran al proceso de sensibilización y empoderamiento comunal en el tema. Los objetivos específicos fueron: en primer lugar, proporcionar un marco conceptual sobre el desarrollo sostenible y la sostenibilidad desde un enfoque tridimensional que permita plantear estrategias de desarrollo, maximizando el uso de recursos disponibles mediante una sensibilización y empoderamiento; en segundo lugar, identificar sus propios criterios respecto a la gestión administrativa y los respectivos procesos efectuados por los diferentes agentes del sector productivo y ambiental del cantón; y, en tercer lugar, formular los procesos de cambio con innovación y creatividad, según las necesidades comunales y de cada agente del sector productivo y ambiental.

La primera sesión estuvo enfocada al tema Principios de desarrollo y sostenibilidad; la segunda sesión abordó la Educación ambiental; la tercera, cuarta y quinta sección enfatizó en la Gestión integral de los residuos sólidos; y la sexta sesión se enfocó en temas de Liderazgo y trabajo colaborativo.

Se compartieron una serie de conocimientos teóricos y prácticos según las necesidades que ellos mismos manifestaron, haciéndolos partícipes en todo momento del proceso de capacitación, lo que generó un sentido de pertinencia y la intención de proponer acciones. Todo lo anterior, con el propósito de estimular la cultura de reducción en la fuente, así como la clasificación, aprovechamiento y disposición final adecuada de los residuos sólidos, con miras a una transformación de la manera de sentir, pensar y actuar del grupo meta respecto a los residuos sólidos y su entorno. 


\section{Fase 6. Aplicación del plan de acción para el mejoramiento de la gestión de residuos sólidos}

En la Conferencia Regional de Educación Superior de América Latina y el Caribe (UNESCOIESALC, 2008) se reivindica el carácter humanista de la educación superior, orientada a la formación integral de personas, ciudadanos y profesionales, capaces de abordar con responsabilidad ética, social y ambiental los retos y participar activa, crítica y constructivamente en la sociedad.

Esta función constitutiva queda latente en la planificación, desarrollo y seguimiento del plan de acción para el mejoramiento de la gestión de residuos sólidos desarrollado en el cantón de Guácimo, cuya área de impacto prioritaria, de esta fase de proyecto, fue el ámbito Educativo - Ambiental, por medio de la generación de procesos de participación e intercambio socioeducativos orientados en esta línea.

La población beneficiaria directa, de esta fase, fueron aproximadamente 150 personas entre niños, niñas, adolescentes, jóvenes, personas adultas y adultos mayores de Santa María de Guácimo.

Para el logro de esta fase se establecieron cinco etapas: a) formación del grupo de facilitadores, b) planificación de las actividades, c) preparación, d) desarrollo y e) evaluación.

Las diferentes sesiones de trabajo con el grupo meta fueron participativas, donde el capacitador fue un facilitador y como tal, promovió la participación, integración, diálogo del grupo, estimulando la empatía, la solidaridad, el compartir de conocimientos, sentimientos, ideas, reflexión personal y profesional, experiencias, recursos e investigaciones.

Como párrafo final de esta sexta fase, cabe justificar este plan de acción acorde con lo que se observa en PLANES (CONARE, 2011), en relación con dos ejes: el eje denominado Gestión que comprende las actividades relacionadas con las dimensiones administrativa y académica, que buscan un desempeño profesional ágil, transparente y que contribuya al mejoramiento de la calidad y la efectividad institucional, y el eje de Pertinencia e Impacto al cual corresponde a la vinculación de las universidades con el entorno, en todas sus dimensiones: social, económica, ambiental y política, por lo tanto, en ese contexto, las instituciones buscarán que sus áreas sustantivas tengan un papel preponderante, innovador y de vanguardia en la propuesta de programas académicos, proyectos de investigación y acciones de desarrollo social en temas estratégicos como es el tema de ambiente.

\section{Fase 7. Divulgación de las diferentes fases del plan de acción}

Como parte del proceso de difusión, se expusieron los resultados en Costa Rica, en seminarios internacionales de investigación durante los años 2012, 2013 y 2014: Simposio Iberoamericano de Ingeniería de Residuos Sólidos, Argentina, año 2013; Congreso de Educación Ambiental, Guatemala, año 2013 y Congreso Internacional de Agronegocios, Italia, año 2014. Además, se han escrito dos artículos. Otros ejemplos de divulgación que se realizaron fueron 
DOI:

URL: http://revistas.ucr.ac.cr/index.php/gestedu

expuestos en Canal 30 (televisora local del cantón), en las redes sociales, en informes anuales y ayudas memorias o actas propias del proceso de investigación y difusión de la información.

\section{Consideraciones finales}

Con el desarrollo de proyectos como el antes referido, se logra enriquecer las actividades sustantivas de las universidades, consolidar y fortalecer las comisiones, programas o espacios de intercambio, la difusión del conocimiento local y del quehacer universitario, el trabajo articulado con el propósito de no generar acciones aisladas, así como el trabajo de manera transversal, que articula los espacios interinstitucionales, tanto en los ámbitos comunales como universitarios.

A nivel de extensión o acción social se logra la integración y accesibilidad al entorno físico, social, económico y cultural, a la salud y la educación, a la información y las comunicaciones comunales, garantizando la inclusión más democrática de los participantes. También la promoción y convocatoria a la participación de los actores sociales de los ámbitos comunales como universitarios; el diseño y gestión de producción de materiales; además de estrategias regionales de investigación, capacitación y formación continua a las personas involucradas en el proceso, en este caso en el área de la educación y la transformación ambiental.

A nivel de la investigación, se logra generar procesos para sistematizar la información focalizada a un área del conocimiento y a una región determinada, lo cual es un insumo para las universidades, las comunidades y los actores sociales. Específicamente para este caso, mediante la investigación de Campos (2014), se elaboró un diagnóstico de línea base sobre la situación de la gestión integral de los residuos sólidos en el cantón; además, se llevó a cabo un estudio sobre conocimientos, habilidades, actitudes y barreras existentes en relación con la gestión de los residuos sólidos; se realizó un estudio de generación y composición de residuos y se diseñó un plan de acción para el mejoramiento de la gestión integral de los residuos sólidos que involucró a los actores sociales del cantón de Guácimo, aspectos que primordialmente se retoman en este artículo.

Al respecto, a nivel general se recomiendan fortalecer los procesos de investigación tanto generados por los docentes con o sin participación estudiantil, así como los trabajos finales de graduación propuestos sobre el tema ambiental y enfocados desde varias perspectivas de acuerdo con las diversas carreras y los diferentes paradigmas, además de las actividades, proyectosy programas de acción social que se realizan de manera universitaria e interuniversitaria con la participación de docentes, estudiantes y personal administrativo desde la extensión docente, extensión cultural y los trabajos comunales universitarios.

En el área sustantiva universitaria de docencia, cabe resaltar que las experiencias teórico prácticas y la aplicación de cada fase del plan de acción sirven como insumo de los diversos procesos académicos universitarios. Como se dijo anteriormente, en la docencia, desde los 
cursos que imparten las universidades, tanto en asignaturas generales o específicas, en las cuales la educación ambiental es un eje central y en algunos casos el eje transversal, también en la aplicación de los principios básicos del cambio social por medio de la cultura ambiental para generar miembros de la comunidad universitaria alfabetizados ambientalmente.

El desarrollo y el fortalecimiento de la Educación Superior constituyen un elemento insustituible para el avance social, la protección ambiental, la sobrevivencia y la promoción de una cultura de paz; de ahí, que la visión y la gestión universitaria deben ser integradoras, capaces de trasformar la realidad hacia adentro y hacia fuera de sus instituciones.

\section{Referencias}

Campos, R. (2014). Actualidad y perspectivas futuras de la Gestión de los Residuos Sólidos en el cantón de Guácimo, Limón, Costa Rica. (Tesis de Doctorado). DOCINADE. TEC-UNA-UNEDUNAM, México.

Campos, R. \& Soto, S. (2014). Análisis de la situación del estado de la GIR en el Cantón de Guácimo, Costa Rica. Tecnología en Marcha, 27(1), 115-124. Recuperado de: file:///C:/Users/equipo\%201/ Downloads/Dialnet-AnalisisDeLaSituacionDelEstadoDeLaGestionIntegralD-4835493.pdf

Caride, J. y Meira, P. (2001). Educación ambiental y desarrollo humano. Barcelona: Ariel.

Chorley, R. J. \& Kennedy, B. A. (1971). Physical Geography: A systems approach. London: PrenticeHall International.

Consejo Nacional de Rectores (CONARE). (2011). Plan nacional de la educación superior universitaria estatal 2011-2015. San José, Costa Rica: CONARE.

Creswell, J. (2005). Educational research. Planing, conducting and evaluating quantitative and qualitative research. Upper saddle river. USA: Pearson Education.

Emmons, K. (1997). Perspectives on Environmental Acting: Reflection and Revision Through Practical Experience. Journal of Environmental Education, 29(1). Recuperado de: http:// www.tandfonline.com/doi/pdf/10.1080/00958969709599105 
Gutiérrez, J. y Pozo, T. (2006). Modelos teóricos contemporáneos y marcos de fundamentación de la educación ambiental para el desarrollo sostenible. Revista Iberoamericana de Educación Ambiental, 41, 21-68. Recuperado de: http://dialnet.unirioja.es/servlet/ articulo?codigo $=2168796$

Guier, E., Rodríguez, M. y Zúñiga, M. (2004). Educación ambiental en Costa Rica: tendencias evolutivas, perspectivas y desafíos. Revista Biocenosis, 18 (1-2). Recuperado de: http:// www.sidalc.net/cgi-bin/wxis.exe/?lsisScript=OET.xis\&method=post\&formato=2\&cantida $\mathrm{d}=1$ \&expresion $=\mathrm{mfn}=035783$

Hungerford, H. R. y Volk, T. (1990). Changing learner behavior through environmental education. Journal of Environmental Education, 21(3). Recuperado de: http://www.tandfonline.com/ doi/abs/10.1080/00958964.1990.10753743?journalCode=vjee20\#.VPpCnvmG9uk

Hsu, S.J.(2004).Theeffects of an environmental education program on responsible environmental behaviour and associated environmental literacy variables in Taiwanese College students. The Journal of Environmental Education, 35(2). Recuperado de: http://www.tandfonline. com/doi/abs/10.3200/JOEE.35.2.37-48\#.VPpCzfmG9uk

Gómez, M. y Reyes, L. (2004). La Educación Ambiental, imprescindible en la formación de nuevas generaciones. Terra Latinoamericana, 22, 515-522. Recuperado de: http://www.redalyc. org/articulo.oa?id=57311096016

Masten, S. E. (2000). Commitment and political governance: why universities, like legislatures, are not organized as firms. Journal of Economics \& Management Strategy, University of Michigan Business Scholl, draft. Recuperado de: http://deepblue.lib.umich.edu/bitstream/ handle/2027.42/71954/j.1530-9134.2006.00113.x.pdf?sequence=1

Martínez, R. y Góngora, N. (2000). Evaluación de la gestión universitaria informe para Comisión Nacional de Evaluación y Acreditación Universitaria (CONEAU). Recuperado de: http://www. coneau.edu.ar/archivos/1326.pdf

López, E., López, C. y Rodríguez, A. (2005). Avances y perspectivas de un modelo de educación ambiental en la zona indígena chontal de Tabasco, México. Recuperado de: http://www. anea.org.mx/eventos/comie2007/Extenso/PonenciaLopezE_RodriguezA_RicaldeC.pdf 
Organización de las Naciones Unidas para la Educación, la Ciencia y la Cultura (UNESCO). (2008). Conferencia regional de educación superior de América Latina y el Caribe (CRES). Colombia: IESALC. Recuperado de: http://www.fvet.uba.ar/institucional/Declaracion.pdf

Organización de las Naciones Unidas para la Educación, la Ciencia y la Cultura (UNESCO) e Instituto de la UNESCO. (2009). Conferencia mundial de la educación superior (CMES): La nueva dinámica de la educación superiory la investigación para el cambio social y el desarrollo. Francia. Recuperado de: http://www.unesco.org/education/WCHE2009/comunicado_es.pdf

Programa Competitividad y Medio Ambiente (CYMA). (2008). Manual para la elaboración de planes municipales de gestión integral de residuos sólidos. Recuperado de: http://ley8839. go.cr/blog/documentos/publicaciones/manual-elaboracion-planes-municipales-girs2008-baja-res.pdf

Programadelas Naciones Unidasparael desarrollo(PNUD)yProyectodeFortalecimientoMunicipal y Descentralización (FOMUDE). (2009). Plan cantonal para el desarrollo humano local de Guácimo. Recuperado de: http://www.ifam.go.cr/PaginalFAM/docs/PRODUCTOS\%20 FOMUDE\%202006-2011/R4-Productos/P23\%20Planes\%20DHL\%20y\%20Agendas\%20 Distritales/Planes\%20de\%20Desarrollo\%20Humano\%20Cantonal/Plan\%20de\%20 Desarrollo\%20Humano\%20Local\%20Cant\%C3\%B3n\%20Gu\%C3\%A1cimo.pdf

Tarrant, M. A. y Cordell, H. K. (1997). The effect of respondent characteristics on general environmental attitude-behavior correspondence. Sage Journal Environment Behavior, 29(5), 618-637. Recuperado de: http://eab.sagepub.com/content/29/5/618.short

Wegener, M. (2000). Spacial models and SIG. New potential and new models (Gisdata). Londres: Taylor and Francis Inc. 